Julian Sepulveda, Agnes Montillet*, Dominique Della Valle, Thanina Amiar, Hubert Ranchon, Catherine Loisel, and Alain Riaublanc

\title{
Experimental determination and modeling of flow curves of xanthan gum solutions over a large range of shear rates
}

https://doi.org/10.1515/arh-2020-0116

Received Jan 05, 2021; accepted Apr 02, 2021

\section{Introduction}

Xanthan gum is one of the hydrocolloids most extensively used in food and non-food applications mainly for viscosity enhancement, emulsion stabilization, temperature stability and pouring properties [3,4]. Xanthan gum solutions present a marked shear thinning behavior $[5,6]$ which is enhanced by the polymer concentration. Depending on the polysaccharide concentration, various concentration regimes can be encountered. In the "dilute regime" there is little or no steric effects and solutions tend to be slightly shear-thinning [7]. Beyond the critical concentration $\left(C^{\star}\right)$, physical interaction between molecules takes place [8] and the system goes into a "semi dilute regime" where the macromolecules start to interact, but internal forces are still dominated by polymer-solvent interactions [9]. Above a second critical concentration value $\left(C^{\star \star}\right)$, the system is in the "concentrated regime" where important entanglements occur and viscosity increases quickly with polymer concentration, leading to a pronounced shear-thinning behavior [7].

Although $C^{\star}$ and $C^{\star \star}$ provide useful information to predict the dilution regime, there are no well-defined values, but rather a range of concentrations where systems gradually change from one concentration regime to another. The calculation of these critical values always implies a certain bias since they depend on the experimental technique employed $[8,10]$. Additionally, in the case of xanthan, a natural polysaccharide, its source, composition and thermal history might as well affect the rheological behaviors of solutions prepared with this polymer [11]. As a matter of fact, for xanthan solutions, different values for $\mathrm{C}^{\star}$ and $\mathrm{C}^{\star \star}$ has been found in the literature. For instance, $\mathrm{C}^{\star}$ is found to

Thanina Amiar, Hubert Ranchon: Formulaction, F-31000 Toulouse, France

Alain Riaublanc: UR 1268 Biopolymères, Interactions, Assemblages, INRAE, T-44000 Nantes, France

\footnotetext{
*Corresponding Author: Agnes Montillet: Université de Nantes, Oniris, CNRS, GEPEA, UMR 6144 F-44000 Nantes, France, France; Email: agnes.montillet@univ-nantes.fr

Julian Sepulveda: Université de Nantes, Oniris, CNRS, GEPEA, UMR 6144 F-44000 Nantes, France, France

Dominique Della Valle, Catherine Loisel: ONIRIS, F-44000 Nantes, France
} 
have values of $0.02 \%$ [12] $0.0126 \%$ [13] $0.007 \%$ [14] while reported values for $\mathrm{C}^{\star \star}$ include $0.078 \%$ [13] and $0.2 \%$ [14], among others.

Visualizing the transitions between the aforementioned regimes is possible by plotting $\log \eta_{s p}$ vs $\log \mathrm{c}[\eta]$, where $\eta_{s p}$ is the specific viscosity, $\eta_{s p}=\eta_{r e l}-1$, c is the concentration and $[\eta]$ is the intrinsic viscosity. It is then possible to notice that the specific viscosity follows a power law as presented in equation $1[15,16]$, where $k$ is a constant and $p$ a parameter that represents the slope having values between 1 and 2 for the dilute regime, 2 to 3 for the semi dilute regime, and 3 to 5 for the concentrated regime [15] The term c $[\eta]$ known as the overlap parameter [17] provides information about the system concentration regime. Generally, at values of $c[\eta]$ between 0.7 and 1.5 the transition from dilute to semi dilute regime is observed and at values of around 6-7 the systems change from semi dilute to a concentrated state [13].

$$
\eta_{s p}=k(c[\eta])^{p}
$$

The intrinsic viscosity $[\eta]$, which provides information on the hydrodynamic volume occupied by the individual isolated polymer molecules [18], depends mainly on the molecular weight and polymer conformation [15]. The models proposed by Huggins [19] (eqn. 2), Kraemer [20] (eqn. 3), Schulz \& Blaschke [21] (eqn. 4) and Tanglertpaibul \& Rao [22] (eqn. 5) are widely used to determine the value of the intrinsic viscosity.

$$
\begin{gathered}
\frac{\eta_{s p}}{c}=[\eta]+k_{1}[\eta]^{2} c \\
\frac{\ln \eta_{s p}}{c}=[\eta]+k_{2}[\eta]^{2} c \\
\frac{\eta_{s p}}{c}=[\eta]+k_{S B}[\eta]^{2} \eta_{s p} \\
1-\frac{1}{\eta_{r e l}}=[\eta] c
\end{gathered}
$$

By extrapolation to a diluted state where $\mathrm{c}$ tends to zero, the second term of the Huggins equation can be neglected [23] and a simplified expression (eqn. 6) allows to easily determine $[\eta]$ as the slope of the plot $\eta_{r e l}$ vs c.

$$
\frac{\eta_{s p}}{c}=[\eta]
$$

The marked shear-thinning behavior of xanthan solutions is of great technological interest as it allows tuning of the rheological properties when dissolved. Products with a high viscosity at rest can be obtained, ensuring a better stability. On the other hand, these solutions exhibit low viscosities when processed under high shear rates facilitating handling of fluid flows and minimizing energy consumption. Characterization of the viscosity profile of xanthan solutions is therefore crucial to design, understand and take advantage of their rheological behavior in industrial processing.

The viscosity of solutions is commonly evaluated by using flow tests and is mainly based on the use of rotational rheometers (also known as shear rheometers). They are usually equipped with several tool geometries, the best suitable choice in each rheological study depending on the properties of fluids and on the desired range of shear stress. The cone-plate is the most common geometry for determining viscoelastic properties of polymeric fluids [24]. For low to medium shear rate ranges $10^{-3}-1000 \mathrm{~s}^{-1}$, this geometry provides reliable data. However, secondary flows may occur beyond a certain shear rate [25], especially since the viscoelastic behavior is pronounced, so that the end of the flow curve does not reflect the viscosity but the inertial stress [26]. The "second Newtonian plateau" observed for the xanthan solutions may often be an artefact.

Mixing, mass transfer or dispersion of immiscible fluids are operations that are actually intensified through the implementation of high shear rates in many industrial units such as rotor-stators [27], extruders [25], spray dryers [28] and high pressure homogenizers. In this kind of processes, encountered shear rates can reach an order of magnitude of around $10^{5} \mathrm{~s}^{-1}$. Even higher shear rates can be reached in innovative applications using microfluidics [25, 29-32].

Ground-breaking techniques are being developed for measuring viscosity at high shear rates using microsystems in laminar straight flow conditions. In some apparatus, as in the Fluidicam ${ }^{\mathrm{TM}}$ used here, the unknown viscosity of a fluid can be deduced from the observation of the coflowing of this fluid with a reference one, using image analysis [33, 34]. The fluid viscosity can also be deduced from a more classical way, based on the measurement of the pressure drop in the channel using pressure sensors [35]. However, due to the small scale of microchannels, these techniques are obviously less suitable and hence less accurate for viscosity measurements at low shear rates. Considering the two methods described above, the lower limit of shear rates available for measurement is about $10^{2}$ $\mathrm{s}^{-1}$.

For characterizations over a very large range of shear rates, i.e. from about $10^{-2} \mathrm{~s}^{-1}$ and up to $10^{5}$ or $10^{6} \mathrm{~s}^{1}$, implementation of complementary characterization techniques is therefore usually needed; the literature is not profuse on this subject. Pipe \& McKinley [36] provided an 
in-depth study on the subject, but, from a technical point of view, their discussion does not cover all of the experimental tasks that experimenters might face. Some studies $[4,35,37-39]$ have reported values of the infinite-shear viscosity $\left(\eta_{\infty}\right)$ of xanthan gum solutions estimated at shear rates higher than $10^{3} \mathrm{~s}^{-1}$. However, there is no discussion over these determinations at high shear rates and even in some cases, some important methodological information is missing. A couple of studies have investigated more exhaustively the characterization of viscosity at high shear rates for both Newtonian and non-Newtonian fluids using modifications of the known parallel plate geometry in conventional rotational rheometry [2, 40-42].

This study aims at extending the knowledge in the field of viscosity characterization of polymeric fluids over a large range of shear rates by exploring two complementary techniques (rotational rheometry and microfluidic rheometry). The case of xanthan gum, a polymer encountered in numerous industrial applications, is selected for this purpose. Solutions from this polymer have been prepared in the concentration range $0.08-0.5 \% \mathrm{w} / \mathrm{w}$ relevant for food applications.

The main objective of this work is to propose a complete methodology to obtain relevant viscosity data in an extended range of shear rates $\left(10^{-3}\right.$ to $\left.10^{5} \mathrm{~s}^{-1}\right)$, in order to model the flow curves in the given range of concentrations. For a given solution, the deviation of the flow curves obtained using the two techniques provides a criterion for the appearance of the secondary flow in the coneplate in terms of a critical value of the Reynolds number. The microfluidic rheometer employed is able to measure the viscosity at sufficiently high shear rates to enable the determination of the second Newtonian plateau by using the Williams-Carreau law. Finally, it is proposed to model the rheological parameters obtained from the WilliamsCarreau fitting as a function of the polymer concentration. In addition, the same approach is applied to xanthan gum solutions with added whey protein isolates (WPI) in order to study the case of a mix of biopolymers that is also of industrial interest.

\section{Materials and methods}

\subsection{Preparation of model solutions}

\subsubsection{Xanthan gum solutions}

A commercial xanthan gum, XG, powder (Ketrol ${ }^{\circledR}$ AP from Kelco) and demineralized water were used for the prepara- tion of the solutions. The powder was added gradually to the solvent at room temperature in a $2 \mathrm{~L}$ mixing vessel under agitation using an overhead stirrer (RZR 2020 from Heidolph instruments). The solutions were formulated at four concentrations $(0.08,0.2,0.4$ and $0.5 \% \mathrm{w} / \mathrm{w})$ commonly encountered in food and non-food applications [3]. During the gradual addition of xanthan gum powder, speed agitation was manually monitored between 100 to $600 \mathrm{rpm}$ in order to avoid air incorporation or formation of lumps. Then, the rotational speed was kept at $300 \mathrm{rpm}$ for 4 hours to achieve complete dissolution and homogenization. No preservative agent was added, and solutions were then stocked at $4^{\circ} \mathrm{C}$ until further characterization. Solutions are named according to the xanthan concentration: $0.08 \%$ (XG008), 0.2\% (XG02), 0.4\% (XG04) and 0.5\% (XG05).

\subsubsection{Whey protein isolates-xanthan gum solutions}

Addition of proteins into a xanthan gum solution is interesting for the tensioactive effect as investigated in a previous study carried out in our laboratory for the manufacturing of food foams using an innovative microfluidic technology [31]. Therefore, solutions containing whey protein isolates (WPI) and xanthan gum (XG) are considered in this study to account for the presence of proteins. The WPI (Pronativ ${ }^{\circledR} 95$ from Lactalis France) was mixed with xanthan gum (Ketrol ${ }^{\circledR}$ AP from Kelco) and then added to demineralized water under agitation following a similar protocol as for the xanthan gum solutions described above. The WPI concentration was set at 3\% w/w while four concentrations were employed for the xanthan gum: 0.2, 0.35, 0.4 and $0.5 \% \mathrm{w} / \mathrm{w}$. Solutions are named according to the concentrations of XG and WPI (WPI3XG02, WPI3XG035, WPI3XG04 and WPI3XG05).

\subsection{Solution viscosity characterization}

Two different rheometric techniques were employed in this study to characterize the viscosity of the solutions over a wide range of shear rates. A rotational rheometer was used to measure the viscosity at low and medium shear rates whereas a microfluidic rheometer from the company Formulaction (France) was used for the viscosity measurements at high shear rates. 


\subsubsection{Rotational rheometer}

A rotational rheometer (AR 1000, TA Instruments) was used with stress-imposed procedures in the range of $10^{-3}$ - $40 \mathrm{~Pa}$ during $5 \mathrm{~min}$ to characterize viscosity in the shear rate range $10^{-3}$ to $10^{3} \mathrm{~s}^{-1}$. The considered duration was beforehand tested to provide time independent results. Two cone-plate geometries were used for the viscosity determination of XG solutions; one having a diameter of $60 \mathrm{~mm}$ with an angle of $2^{\circ}(60-2)$ and another one having a diameter of $40 \mathrm{~mm}$ and an angle of $4^{\circ}$ (40-4). WPIXG solutions are only characterized with the geometry 40-4. All samples were tested at $20^{\circ} \mathrm{C}$ and measurements were made in triplicate.

\subsubsection{Microfluidic rheometer}

A microfluidic rheometer (FLUIDICAM RHEO ${ }^{\mathrm{TM}}$ from Formulaction, France) was used for viscosity determinations at high shear rates $\left(>10^{3} \mathrm{~s}^{-1}\right)$. The principle of this device is based on establishing a cocurrent fully developed laminar flow (Figure 1) between the studied fluid (sample fluid) and a Newtonian reference fluid whose viscosity is known. The relative section occupied by the fluids is proportional to the product of the viscosity ratio and the flowrate ratio (eqn. 7) [33, 34]. $W$ represents the width of the channel occupied by the fluid, $\eta$ the viscosity and $Q$ the flowrate. The subscript $R$ stands for the reference fluid. The viscosity of the fluid under study can be then determined by knowing the interface position which is measured by a camera and image analysis. Flowrates are carefully controlled by syringe pumps and due to the small length scales in the mi-

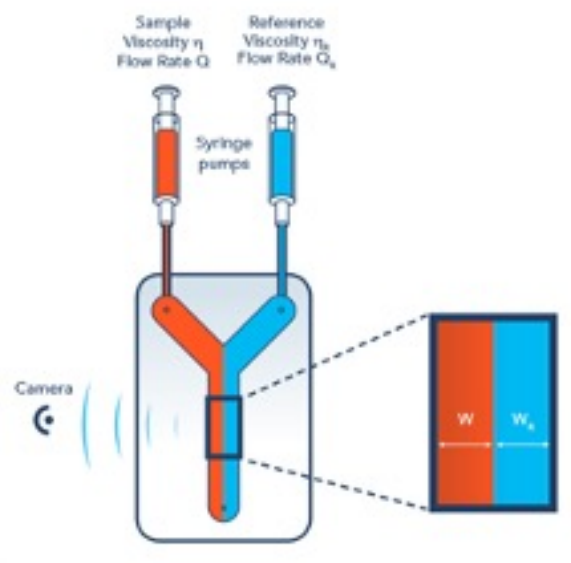

Figure 1: Schematic representation of the laminar co-flow of two fluids inside the FLUIDICAM RHEO ${ }^{\mathrm{TM}}$ microfluidic rheometer. crodevice, high shear rates can be easily attained while ensuring laminar co-flow. Two complementary microchips of varying dimensions were used for the measurements of the XG solutions viscosities. These devices have a rectangular geometry (Figure 1) with a width of $2.2 \mathrm{~mm}$, and two different depths of the channel (50 and $150 \mu \mathrm{m}$ ). The microchip with a channel depth of $150 \mu \mathrm{m}$ generally allows viscosity determinations in the range of shear rates (100 - 18000$)$ $\mathrm{s}^{-1}$ while the device having a channel depth of $50 \mu \mathrm{m}$ allows viscosity measurements in the range of shear rates $\left(2000-2 \times 10^{5}\right) \mathrm{s}^{-1}$. In this work, the viscosities of WPIXG solutions were measured only with the device having a channel depth of $50 \mu \mathrm{m}$, while both devices (150 and 50 $\mu \mathrm{m}$ ) were used for measurements of the XG solutions. The reference fluid is a solution of polyethylene glycol (PEG) having a viscosity of $6.87 \mathrm{mPa} \cdot \mathrm{s}$ at $20^{\circ} \mathrm{C}$ in the FLUIDICAM $\mathrm{RHEO}^{\mathrm{TM}}$. For each flowrate condition, the location of the interface between the two fluids is measured by a camera. In stationary conditions, six to ten pictures are captured to make a flow curve point.

The solution apparent viscosity was deduced as follows (equation 7):

$$
\frac{W}{W_{R}}=\frac{\eta_{\text {app }}}{\eta_{R}} \frac{Q}{Q_{R}}
$$

In order to determine the true viscosity $\left(\eta_{\text {true }}\right)$ from the apparent viscosity $\left(\eta_{\text {app }}\right)$ obtained from equation 7 , equation 8 is used.

$$
\tau=\eta_{\text {app }} \dot{\gamma}_{\text {app }}=\eta_{\text {true }} \dot{\gamma}_{\text {true }}
$$

As the channel height is small compared to channel width, as well as to the respective widths occupied by the two fluids, the apparent shear rates in laminar flows can be estimated as in a 2D flow (eqn. 9):

$$
\dot{\gamma}_{\text {app }}=\frac{(6 Q)}{\left(w h^{2}\right)}
$$

where $Q$ is the volume flow rate of the concerned fluid, $h$ the height of the channel and $w$ the width occupied by the fluid [43].

The shear stress $(\tau)$ was determined using the apparent viscosity and apparent shear rates measured during experimentation. The true shear rate $\left(\dot{\gamma}_{\text {true }}\right)$ in a rectangular duct was then calculated from the WeissenbergRabinowitsch-Mooney equation [44]. The term $\frac{d \ln \dot{\gamma}_{a p p}}{d \ln \tau}$ corresponds to the slope of the plot $\ln \dot{\gamma}_{\text {app }}$ vs $\ln \tau$. Finally, the true viscosity was obtained from equation (10):

$$
\dot{\gamma}_{\text {true }}=\frac{\dot{\gamma}_{\text {app }}}{3}\left[2+\frac{d \ln \dot{\gamma}_{\text {app }}}{d \ln \tau}\right]
$$




\subsection{Modeling the solutions viscosity and correlation with polymer concentration}

\subsubsection{Determination of a critical Reynolds number $\operatorname{Re}_{c}$ using a cone-plate geometry}

It is well known that due to centrifugal forces, flow recirculations (namely secondary flow or Taylor vortices) may take place when using a coneplate geometry. The concern is to have a quantitative criterion for the onset of these instabilities that supports the validity of the measurements. Several authors have discussed this problem either using empirical means or by developing analytic or numerical calculations [1, 45-47]. However, there is still no universal simple way to predict the onset of instabilities for any fluid and any cone-plate geometry as easily as is in the Couette flow. Therefore, in the present study, we have developed a simple semi-empirical methodology to identify the region of shear rates where instabilities are likely to appear when using the cone-plate geometry. To give more weight to this approach, by analogy with the Taylor number for Couette flow, a rotational Reynolds number is computed to characterize the limit of the onset of instabilities for the different combinations of geometries and solutions.

This conventional Reynolds number is defined for rotational devices used in process engineering [48], like stirred vessels; it is designated as 'rotational Reynolds number' in the following:

$$
R e=\frac{\rho N D^{2}}{\eta}
$$

Here $\rho$ is the solution density, $D$ the diameter of the cone geometry, and $N$ the rotational speed, all variables are in International System of Units (SI). In rotational flows, the Reynolds number is an internal measure of the ratio between centrifugal and viscous forces. One can notice that it is very similar to the Taylor number and can be considered as that for a given geometry. Reynolds numbers based on centrifugal forces were previously used by several authors like Turian [1] who developed analytical solutions for Newtonian and non-Newtonian flows in cone-plate systems and Fewel and Helluns [45] who characterized secondary flows of Newtonian fluids in cone-plate viscosimeters.

The rotational critical Reynolds number $\left(\operatorname{Re}_{c}\right)$ (eqn. 13) and the critical rotational speed defined by equation 12 were determined by the shear rate $\dot{\gamma}_{\text {max }}$ where the rheometer and the Fluidicam ${ }^{\mathrm{TM}}$ flow curve diverged. Equation 12 derives from the calculation of the uniform shear rate in the cone-plate flow.

$$
N_{c}=\frac{\dot{\gamma}_{\max } \theta}{2 \pi}
$$

$$
\operatorname{Re}_{c}=\frac{\rho N_{c} D^{2}}{\eta}
$$

The two datasets (rotational rheometer and microfluidic rheometer) were plotted on a same graph of viscosity vs shear rate and each one fitted by the Williams-Carreau law given in equation 14, where $\eta$ stands for the fluid viscosity at the current shear rate $\dot{\gamma}, \eta_{0}$ is the zero-shear viscosity (first plateau), $\eta_{\infty}$ the infinite shear-viscosity (second plateau), $m$ is a constant related to the flow index, and $\lambda$ is a time constant representing the shear rate where the rheological behavior changes from the $1^{\text {st }}$ Newtonian plateau to the shear-thinning range.

$$
\frac{\eta-\eta_{0}}{\eta_{0}-\eta_{\infty}}=\frac{1}{\left(1+(\lambda \dot{\gamma})^{2}\right)^{\frac{m}{2}}}
$$

The diverging region between the two flow curves determines the maximum shear rate $\dot{\gamma}_{\max }$ beyond which flow instabilities are likely to appear due to the centrifugal forces. The maximum shear rate was estimated at the point where the two models diverged by more than $10 \%$. An example of this approach is given in Figure 2, for $0.08 \% \mathrm{w} / \mathrm{w}$ xanthan gum solution and the 60-2 cone-plate geometry.

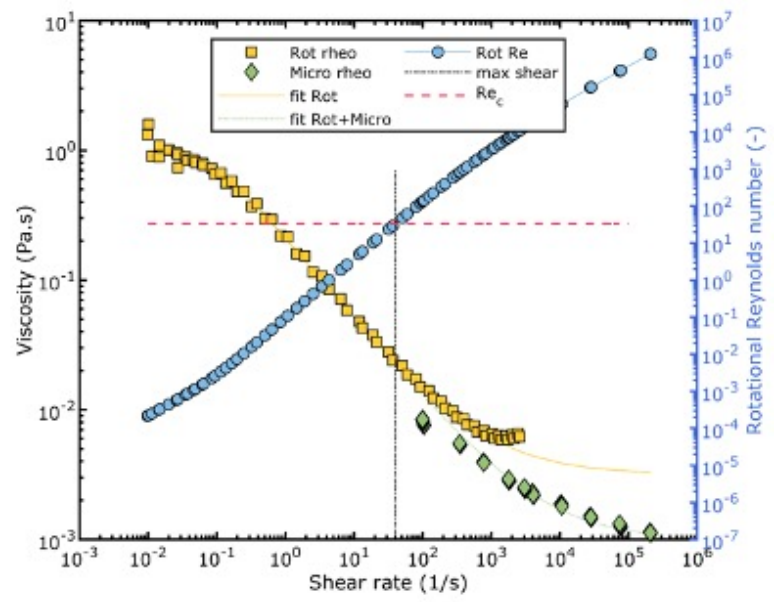

Figure 2: Determination of the onset of inertial effects for xanthan gum solution $0.08 \% \mathrm{w} / \mathrm{w}$ using the cone-plate geometry $60-2$ $\left(60 \mathrm{~mm}\right.$ diameter and $2^{\circ}$ angle). In legend: viscosity measurements from rotational rheometer (Rot rheo), viscosity measurements obtained with microfluidic rheometer (Micro rheo), model fit for data from rotational rheometer (fit Rot), model fit for data from microfluidic rheometer (fit Rot+Micro), calculated rotational Reynolds numbers (Rot Re), shear rate where instabilities begin to appear (max shear) and calculated critical Reynolds number $\left(\operatorname{Re}_{c}\right)$. 


\subsubsection{Determination of the fitting rheological parameters of the Williams-Carreau law}

In a first step, rheological parameters were determined from the rotational rheometer flow curve. Then, viscosity values above the calculated $\operatorname{Re}_{c}$ were replaced by the Fluidicam $^{\mathrm{TM}}$ data so as to determine a model for the whole range of shear rates: in this approach, $\eta_{0}, \lambda, m$, were kept constant from the first step, and only the second plateau $\eta_{\infty}$ was fitted in the second step.

\subsubsection{Modeling the fitted rheological parameters of the Williams-Carreau law with polymer concentration}

The fitted parameters from the Williams-Carreau model $\left(\eta_{0}, \lambda, m\right.$, and $\left.\eta_{\infty}\right)$ determined as explained in section 2.3.2 were then correlated with the polymer concentration. For the first Newtonian plateau the viscosity $\eta_{0}$ was fitted to a power-law model (eqn. 15) as presented in $[15,16]$. From the literature, the intrinsic viscosity $[\eta]_{0}$ for xanthan gum solutions in water is approximately $15.5 \mathrm{~m}^{3} \mathrm{~kg}^{-1}$ [18]. In equation 15 , the specific viscosity $\eta_{s p 0}$ is built on the first plateau viscosity.

$$
\eta_{s p 0}=k\left(c[\eta]_{0}\right)^{p}
$$

For the second Newtonian plateau $\eta_{\infty}$, a linear trend as a function of concentration was observed (eqn. 16). Therefore, an attempt was made to determine an intrinsic viscosity $[\eta]_{\infty}$ corresponding to the conformation equilibrium at the second plateau. The specific viscosity in this case is built on the infinite-shear viscosity. The model proposed by Huggins [19] when concentration tends to zero reads:

$$
\frac{\eta_{s p_{\infty}}}{c}=[\eta]_{\infty}
$$

For the parameters $\lambda$ and $m$, no theoretical groundings were used and these values were fitted with empirical models to evaluate their variation as function of the polymer concentration.

\section{Results and Discussion}

\subsection{Determination of the critical Reynolds number}

The rotational critical Reynolds number $\left(R e_{c}\right)$ is estimated at the onset of flow instabilities due to centrifugal forces in the rotational rheometer. As explained in the methodology, the shear rate $\dot{\gamma}_{\max }$ determining the onset of these instabilities is estimated experimentally where the two model fits (Figure 2) deviate by more than $10 \%$. Then, a critical Reynolds number $\operatorname{Re}_{c}$ can be computed using equations 12 and 13. This procedure is carried out for all solutions and geometries tested. It is important to recall that the viscosity at high shear rates is characterized with the same microfluidic geometry described in the methodology while two different cone-plate geometries (60-2 and 40-4) are used for the characterization at low to medium shear rates. Table 1 summarizes the values of $\dot{\gamma}_{\max }$ and $R e_{c}$ found in this study for all the experiments. The $R e_{c}$ is dependent on the cone-plate geometry used, with higher values (33-36) for the XG solutions using the geometry 60-2, compared to those obtained with the geometry 40-4 (4-10) for the same solutions. The higher value of $R e_{c}$ obtained with the ge-

Table 1: Estimated maximum shear rate $\dot{\gamma}_{\max }$ and critical Reynolds number $\left(R e_{c}\right)$ for all experimental conditions.

\begin{tabular}{cccccc}
\hline Solution & Geometry & $\begin{array}{c}X G \\
\% w / w\end{array}$ & $\begin{array}{c}W P I \\
\% w / w\end{array}$ & $\begin{array}{c}\dot{\gamma}_{\max } \\
s^{-1}\end{array}$ & $\begin{array}{c}\operatorname{Re}_{c} \\
-\end{array}$ \\
\hline XG008 & $60-2$ & 0.8 & 0 & 40 & 33 \\
XG02 & $60-2$ & 2 & 0 & 88 & 36 \\
XG04 & $60-2$ & 4 & 0 & 140 & 35 \\
XG05 & $60-2$ & 5 & 0 & 149 & 33 \\
XG008 & $40-4$ & 0.8 & 0 & 12 & 4 \\
XG04 & $40-4$ & 4 & 0 & 81 & 10 \\
XG05 & $40-4$ & 5 & 0 & 76 & 8 \\
WPI3XG02 & $40-4$ & 2 & 3 & 48 & 12 \\
WPI3XG035 & $40-4$ & 3.5 & 3 & 88 & 11 \\
WPI3XG04 & $40-4$ & 4 & 3 & 88 & 12 \\
WPI3XG05 & $40-4$ & 5 & 3 & & 104 \\
\hline
\end{tabular}




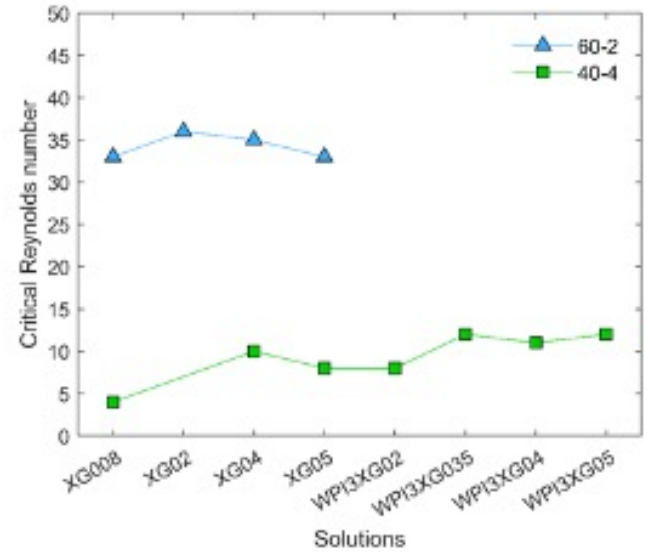

Figure 3: Critical Reynolds numbers for the two cone-plate geometries.

ometry 60-2 would be the result of a greater diameter leading to more intense centrifugal forces. It is also noticeable how the $R e_{c}$ for WPIXG solutions with geometry 40-4 are very close to the values found for XG solutions with the same geometry. This is demonstrating that the rotational Reynolds number, with the meaning of a Taylor number, provides a relevant universal parameter through its critical value $R e_{c}$ to predict the inertial transition for a given geometry. Figure 3 presents the rotational critical Reynolds numbers identified for the appearance of secondary flows for the experimental conditions used in this study. The critical Reynolds numbers identified at rather low shear rates (under $150 \mathrm{~s}^{-1}$ ) are in fair agreement with the literature [26]. The works of Heuser \& Krause and that of Sdougos et al. $[49,50]$ have studied the local flow structure in a cone-plane geometry and have found that secondary flows might appear at Reynolds numbers as low as 4. With regard to polymer fluids, Kulicke \& Porter [51] have reported that these flow instabilities might appear from shear rates as low as $5 \mathrm{~s}^{-1}$. Similarly, Davies \& stokes [2] have reported a Reynolds number of 100 as the limit where the effects of secondary flows are significant. For Newtonian fluids, it has been reported that the inertial disturbances caused by these secondary flows might appear at Reynolds numbers 800 [46] for cone angle between $1^{\circ}$ and $4^{\circ}$, which is higher than other results, but it is clear that the viscoelastic behavior plays an important part in this transition.

\subsection{Determination of the fitting rheological parameters of the Williams-Carreau law}

\subsubsection{General considerations about flow curves}

After elimination of the data from the rotational rheometer that are above of the rotational critical Reynolds number, the viscosities of the solutions are modeled over approximately eight decades $\left(10^{-3}\right.$ to $\left.10^{6} \mathrm{~s}^{-1}\right)$. The Figure 4 presents the flow curves (markers) and corresponding models (solid lines) for the XG solutions for both coneplate geometries, 60-2 (Figure 4a) and 40-4 (Figure 4b) as well as for WPIXG solutions with the geometry 40-4 (Figure 4c). The model fitting parameters for all experimental conditions are summarized in Table 2. Figure 4a displays the flow curves for XG solutions at four different concentrations $(0.08 \%, 0.2 \%, 0.4 \%$, and $0.5 \% \mathrm{w} / \mathrm{w})$. The marked shear-thinning behavior of the xanthan solutions [39, 52] is clear for the four cases, around four orders of magnitude for the viscosity loss. For the most concentrated so-

Table 2: Williams-Carreau fitted rheological parameters for all solutions. MADP is the mean absolute percent deviation between experimental data and model.

\begin{tabular}{ccccccc}
\hline Solution & Geometry & $\begin{array}{c}\eta_{0} \\
\mathrm{~Pa} \cdot \mathrm{s}\end{array}$ & $\begin{array}{c}\eta_{\infty} \\
\times 10^{-3} \mathrm{~Pa} \cdot \mathrm{s}\end{array}$ & $\begin{array}{c}\lambda \\
\mathrm{s}\end{array}$ & $\begin{array}{c}m \\
-\end{array}$ & $\begin{array}{c}\text { MAPD } \\
\%\end{array}$ \\
\hline XG008 & $60-2$ & 0.8 & 1.1 & 9.48 & 0.60 & 11.5 \\
XG02 & $60-2$ & 11.5 & 1.5 & 15.4 & 0.79 & 8.46 \\
XG04 & $60-2$ & 84.2 & 2.2 & 29.4 & 0.87 & 12.1 \\
XG05 & $60-2$ & 146 & 2.3 & 34.8 & 0.87 & 12.7 \\
XG008 & $40-4$ & 1.2 & 1.1 & 14 & 0.63 & 8.3 \\
XG04 & $40-4$ & 110 & 2.0 & 32 & 0.86 & 17 \\
XG05 & $40-4$ & 182 & 2.5 & 42.1 & 0.87 & 14.7 \\
WPI3XG02 & $40-4$ & 20.4 & 1.3 & 20.7 & 0.78 & 4.7 \\
WPI3XG035 & $40-4$ & 95 & 2.0 & 32.6 & 0.84 & 4.7 \\
WPI3XG04 & $40-4$ & 125 & 2.0 & 35.3 & 0.82 & 6.4 \\
WPI3XG05 & $40-4$ & 201 & 2.1 & 41.7 & 0.85 & 6 \\
\hline
\end{tabular}



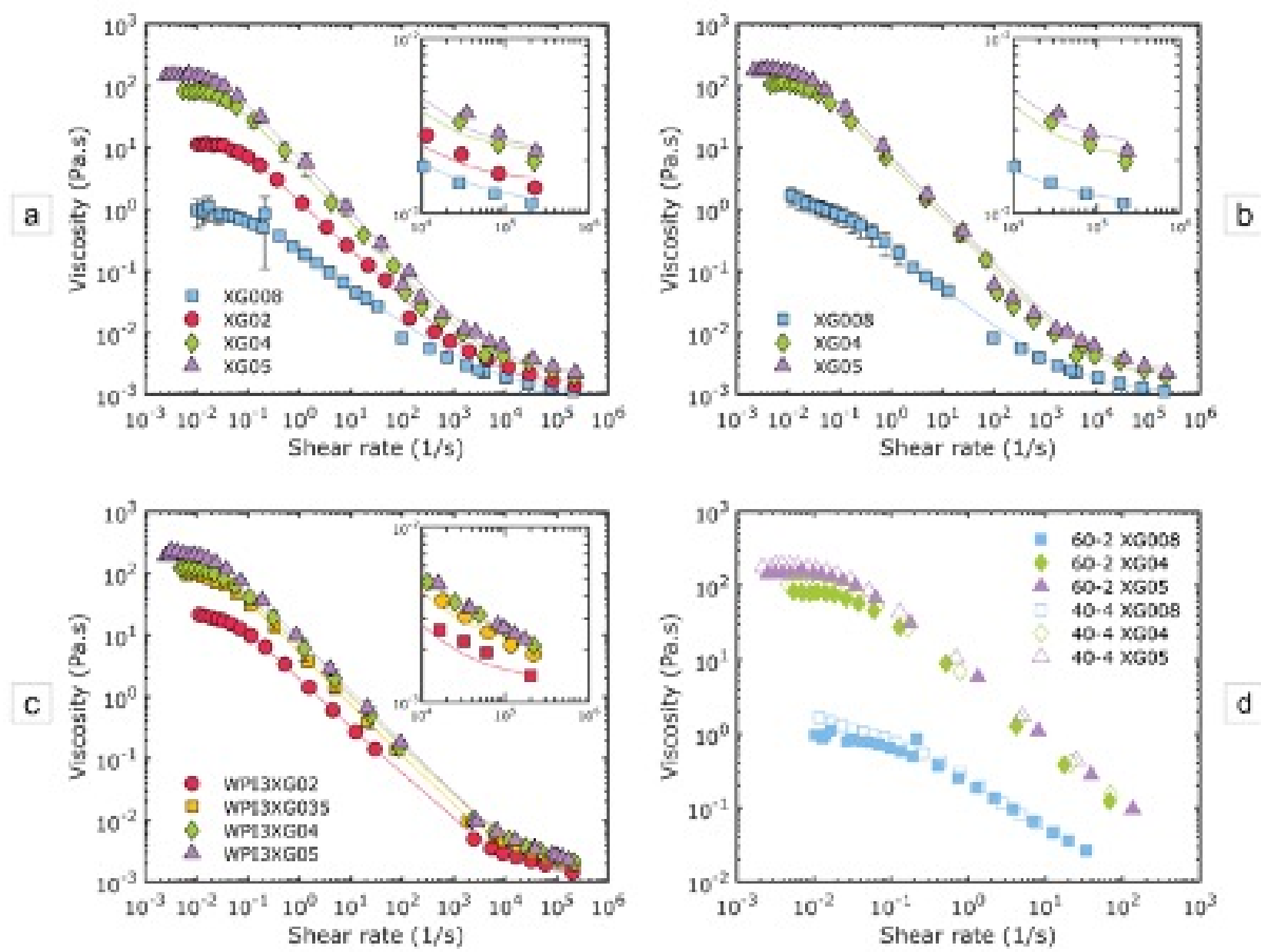

Figure 4: Flow curves and model fits. a) 60-2 cone-plate geometry + microfluidic rheometer for XG solutions. b) 40-4 cone-plate geometry + microfluidic rheometer for XG solutions. c) 40-4 cone-plate geometry + microfluidic rheometer for WPIXG solutions. d) comparison of viscosity data from rotational rheometer using cone-plate geometries 60-2 and 40-4 for XG solutions.

lutions (XG04 and XG05), a more structured polymer network allows an enhanced viscosity at rest as argued by Narchi et al. [53]. Under shear, the complex polymer network is disrupted [4] and disentanglement and reorientation of the semi-rigid polymer chains contribute to the shear-thinning behaviors observed [15, 54, 55]. Destructuring under stress continues until no more conformational changes are possible; this new equilibrium is reflected by a constant viscosity, namely the second Newtonian plateau. In Figure 4, this second Newtonian plateau $\left(\eta_{\infty}\right)$ seems to occur at shear rates around $10^{5}$ to $10^{6} \mathrm{~s}^{-1}$.

\subsubsection{Discussion on the second plateau viscosity values}

The most delicate point in this study is the relevant determination of the second Newtonian plateau as it is the base of a fair prediction of the viscosity at high shear rates. Some comparisons of our results and previous literature studies are worth to be discussed here, despite the fact that the polymer origin may have an impact on the viscosity values. It is commonly admitted that the viscosity of this second plateau is close to that of the solvent (here water at $20^{\circ} \mathrm{C}$ being $\left.10^{-3} \mathrm{~Pa} \cdot \mathrm{s}\right)$. Our actual values allow to qualify this feature more precisely.

Comba et al. [37] have reported a value of $\eta_{\infty}$ of 0.0020 $\mathrm{Pa} \cdot \mathrm{s}$ for a XG solution having a concentration of $3 \mathrm{~g} \cdot \mathrm{L}^{-1}$ $(\sim 0.3 \%$ wt.). This value seems to fit quite well the values found in this study for XG02 (0.2\% wt.) and XG04 (0.4\% 
wt.) which were in the range $(0.0015-0.0022) \mathrm{Pa} \cdot \mathrm{s}$. These authors also used two techniques to model the viscosity over a wide range of shear rates. For the evaluations at high shear rates, they succeeded in evaluating viscosity with the parallel plate geometry with small gaps for the solution with concentration $3 \mathrm{~g} \cdot \mathrm{L}^{-1}$, but this might not be possible for more diluted solutions. Xu et al. [38] also modeled the viscosity of XG solutions at three different concentrations (0.1, 0.25 and $0.5 \%$ wt.). For the concentration $0.5 \%$, the authors obtained a $\eta_{\infty}$ of $0.004 \mathrm{~Pa} \cdot \mathrm{s}$ which is almost two times more than the one found in this study ( 0.0023 $\mathrm{Pa} \cdot \mathrm{s})$ for the same concentration. They used only a rotational rheometer and the characterizations were done at $20^{\circ} \mathrm{C}$, over a shear rate range from 0.001 to $1000 \mathrm{~s}^{-1}$. However, they do not specify the type of geometry used. Another interesting example of the determination of the second Newtonian plateau found in the literature is the study from Mrokowska \& Krzton-Maziopa [39]. In this case, the authors used coaxial cylinders, at $21^{\circ} \mathrm{C}$ and a shear rate range between $10^{-2}$ and $10^{3} \mathrm{~s}^{1}$ for the generation of flow curves of $\mathrm{XG}$ solutions having concentrations in the range $(0.25-1.4) \mathrm{g} \cdot \mathrm{L}^{-1}(\sim 0.025-0.14 \%$ wt.). The infinite shear viscosity $(0.00413 \mathrm{~Pa} \cdot \mathrm{s})$ of their solution at $0.075 \%$ is two times higher when compared with the one $(0.023 \mathrm{~Pa} \cdot \mathrm{s})$ reported here for the solution XG008 $(0.08 \%)$. Similarly, for a $0.4 \%$ XG solution, Wyatt \& Liberatore [14] found a $\eta_{\infty}$ of $0.0051 \mathrm{~Pa} \cdot \mathrm{s}$ which is much higher than the value, 0.0022 $\mathrm{Pa} \cdot \mathrm{s}$, found here for a XG solution at the same concentration (XG04). In this case, the authors used two geometries (cone-plate $60 \mathrm{~mm}-1^{\circ}$ and concentric cylinders) for the rheological characterizations of their solutions without specifying which one is used for each solution. The fitting parameters were calculated using a shorter shear rate range $10^{3}$ to $10^{3} \mathrm{~s}^{-1}$ which might explain the higher values of $\eta_{\infty}$ predicted in their study.

From the above discussion it is clear that important methodological information is missing for the characterization of viscosity of xanthan gum solutions at high shear rates. In addition, it is noteworthy that reported values for $\eta_{\infty}$ using only traditional rotational rheometric techniques (irrespective of the geometry), are generally twice as large as those found in this study. Therefore, the determinations of the infinite-shear viscosity presented above, done exclusively with data obtained from a rotational rheometer without explicitly considering inertial instabilities, might not be accurately characterizing $\eta_{\infty}$. Nonetheless, recent works $[2,40]$ are investigating the possibility of extending the range of measurable shear rates using a modified rotational rheometer. This can lead to improvements in the accuracy of high shear rates viscosity determinations for polymer fluids using traditional rheometric techniques. The emerging field of microfluidics is offering an interesting alternative for rheological characterizations at high shear rates. A previous study that explored viscosity characterizations at high shear rates by microfluidic rheometry has been undertaken by Pipe et al. [36]. In their research, these authors were able to model the viscosity of a $0.3 \% \mathrm{XG}$ solution over the shear rate range $10^{-3}-10^{4} \mathrm{~s}^{-1}$ at $22^{\circ} \mathrm{C}$ using two conventional geometries $(40 \mathrm{~mm}$ parallel plates and $60 \mathrm{~mm}$ cone-plate) and a microfluidic capillary viscometer. The infinite shear viscosity for this solution was around $0.002 \mathrm{~Pa} \cdot \mathrm{s}$ which is also in agreement with the range of $\eta_{\infty}$ found in our study between solutions at similar concentrations XG02 (0.0015 Pa·s) and XG04 (0.0022 $\mathrm{Pa} \cdot \mathrm{s})$.

\subsubsection{Effect of WPI protein addition}

A comparison between XG and WPIXG solutions can be proposed thanks to the measurements performed with the geometry 40-4 (Figure 4b and 4c). It is noticeable that the addition of another polymer, in this case WPI, significantly increases the value of $\eta_{0}$ while differences are less for $\eta_{\infty}$. The increase of the solutions zero-shear viscosity when adding WPI stems from a more important phenomenon of steric hindrance between the two polymers. No interaction between the polymers is presumed since no interaction have been identified at WPI concentrations $\leq 4 \% \mathrm{wt}$. and $\mathrm{XG} \leq 0.5 \%$ wt. [56]. Additionally, it has been reported a low interaction of native WPI with other molecules in solution unless they undergo conformational changes, for instance due to heating $[57,58]$.

\subsubsection{Effect of the cone-plate geometry}

Our data allow to compare the results for the same solutions XG008, XG04 and XG05 for the two cone-plate systems, (see Figures 4a, 4b, 4d and Table 2). Overall, there are no significant discrepancies except for the values of $\eta_{0}$. The zero-shear viscosity values are systematically lower for the geometry 60-2. This viscosity reduction with a small gap has already been observed in other studies for parallel plate geometry [2]. Acording to Kamykowski [59], the low shear rates measurement are less affected by the torque limit with the 60-2 system than the 40-4 system, and we consider that the measurements obtained with the greater diameter and smaller angle are more reliable for the final modeling. Nevertheless, the 40-4 measurements are treated in the next section of the study for comparison purposes. 
Table 3: Model equations and fitted parameters for the correlations of the rheological parameters with polymer concentration. Adjustment of models in terms of MAPD (mean absolute percent deviation).

\begin{tabular}{|c|c|c|c|c|}
\hline \multirow{2}{*}{$\begin{array}{l}\text { Rheological } \\
\text { parameters }\end{array}$} & \multirow{2}{*}{ Equation } & \multicolumn{3}{|c|}{ Fitted parameters and model error } \\
\hline & & $X G-60-2^{\circ}$ & $X G-40-4^{\circ}$ & WPIXG-40-4 ${ }^{\circ}$ \\
\hline \multirow{3}{*}{$\eta_{0}$} & \multirow{3}{*}{$\eta_{0}=\left(k(c[\eta])^{p}+1\right) \eta_{\text {solvent }}$} & $\mathrm{k}=0.61$ & $\mathrm{k}=1.19$ & $k=2.54$ \\
\hline & & $p=2.86$ & $p=2.74$ & $p=2.62$ \\
\hline & & $\mathrm{MAPD}=2.76$ & $\mathrm{MAPD}=3.37$ & MAPD $=4.74$ \\
\hline \multirow[b]{2}{*}{$\eta_{\infty}$} & \multirow{2}{*}{$\eta_{\infty}=(c[\eta]+1) \eta_{\text {solvent }}$} & {$[\eta]=0.26$} & {$[\eta]=0.26$} & {$[\eta]=0.22$} \\
\hline & & MAPD $=5.36$ & MAPD $=5.63$ & MAPD $=8.11$ \\
\hline \multirow{3}{*}{$\mathrm{m}$} & \multirow{3}{*}{$m=a c^{b}$} & $a=0.64$ & $a=0.66$ & $\mathrm{a}=0.73$ \\
\hline & & $\mathrm{b}=0.21$ & $b=0.18$ & $b=0.09$ \\
\hline & & $\mathrm{MAPD}=3.13$ & MAPD $=1.09$ & MAPD $=1.02$ \\
\hline \multirow{3}{*}{$\lambda$} & \multirow{3}{*}{$\lambda=a c+b$} & $a=6.20$ & $a=6.45$ & $a=7.02$ \\
\hline & & $b=3.96$ & $b=8.34$ & $b=7.13$ \\
\hline & & $M A P D=3.74$ & MAPD $=4.58$ & MAPD $=1.65$ \\
\hline
\end{tabular}
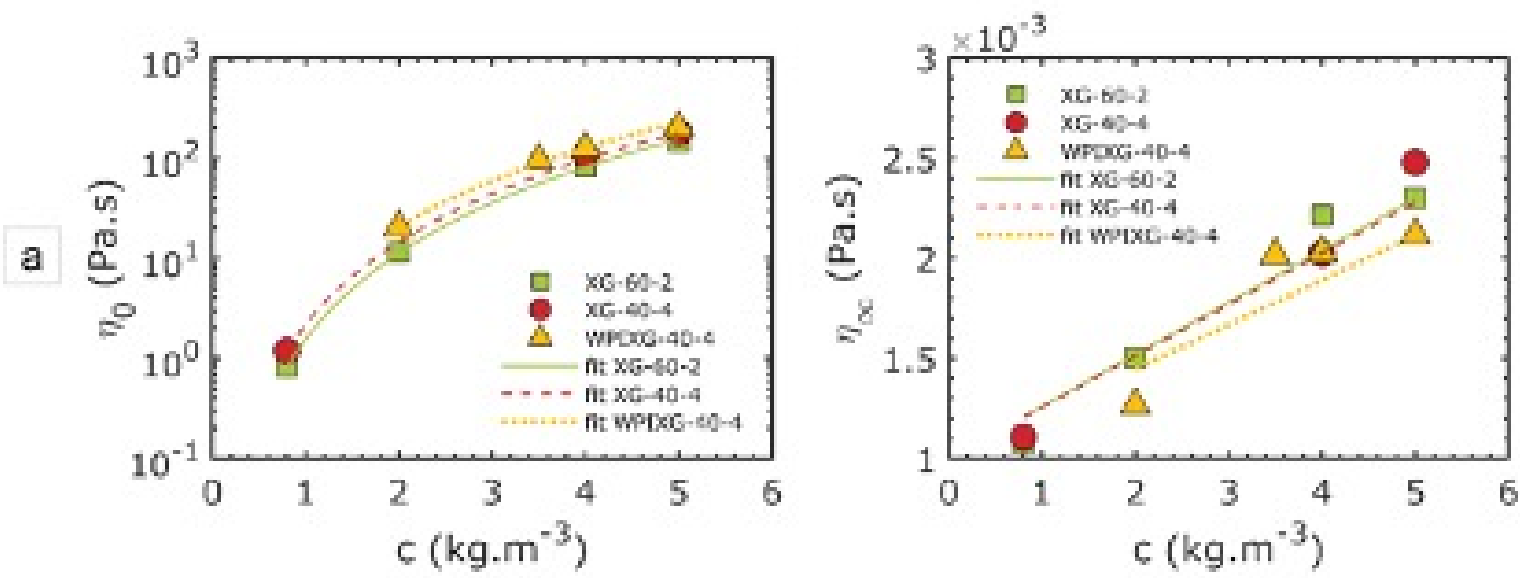

b
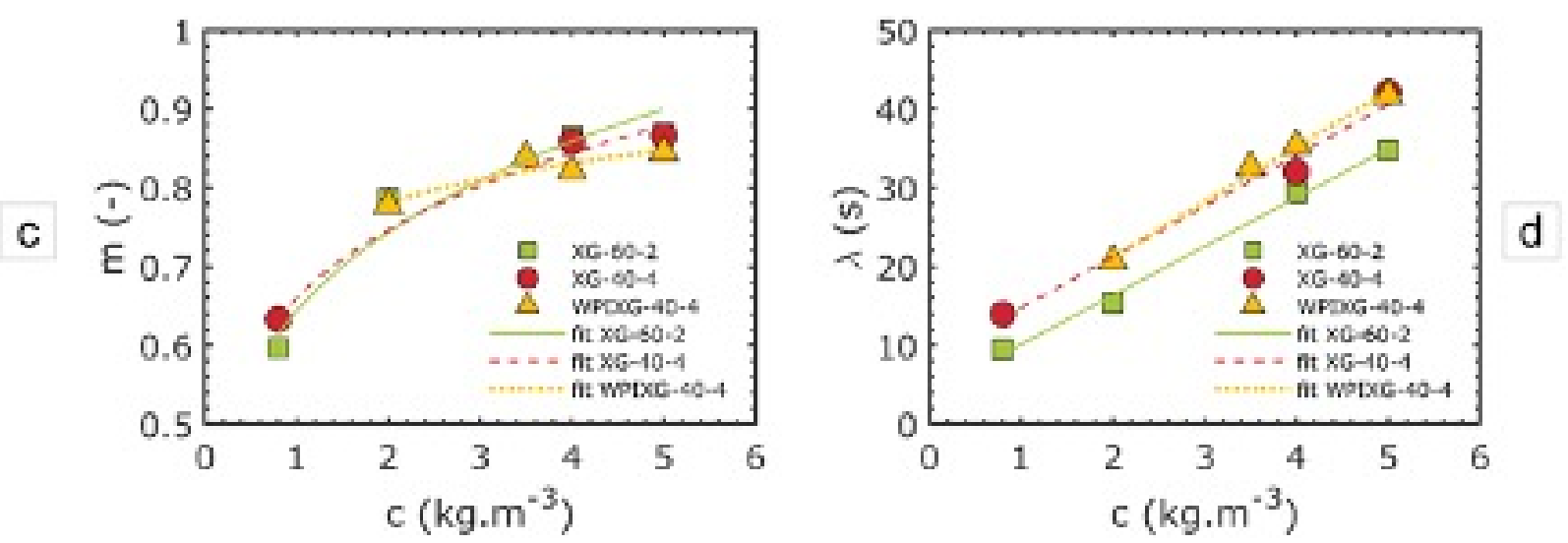

Figure 5: Correlation of the rheological parameters of the Williams-Carreau model with polymer concentration a) zero-shear viscosity ( $\left.\eta_{0}\right)$ b) infinite-shear viscosity $\left(\eta_{\infty}\right)$ c) constant related to flow index $(m)$ d) time constant $(\lambda)$. 


\subsection{Correlating the fitted rheological parameters with polymer concentration}

The rheological fitted parameters found for each solution presented in the previous section are now modeled as a function of concentration. Figure 5 presents the modeling of $\eta_{0}, \eta_{\infty}, m$ and $\lambda$ for all experimental conditions, and the equation values are recapitulated in Table 3. Model adjustment is evaluated in terms of the mean absolute deviation (MAPD) given in percent.

\subsubsection{The first Newtonian plateau viscosity}

For the modeling of the first Newtonian plateau $\eta_{0}$, the equation 15 presented in section 2.3.3 is employed; the purpose here is to use a well-known equation type to model the zero-shear viscosity $\eta_{0}$ in the range of concentration of our interest and see if errors are acceptable to use this model as a predictive tool for future studies. Equation 15 is handled with the solvent viscosity (water) of $10^{-3} \mathrm{~Pa} \cdot \mathrm{s}$, and the intrinsic viscosity $[\eta]_{0}$ of xanthan gum in water taken at $15.5 \mathrm{~m}^{3} \mathrm{~kg}^{-1}$ [18]. The values of the constants $k$ and $p$ are fitted with a solver, leading to a deviation less than $5 \%($ MAPD $\leq 5 \%)$. The results are presented in Table 3 and displayed in Figure 5 .

The $k$ value is increasing with addition of WPI, while for the constant $p$, similar values are obtained in the range $2.6-2.9$ (percent differences $<10 \%$ ) denoting the end of the semi entangled regime and the beginning of the concentrated regime as stated by Roy [15]. To better visualize the dilution regime of our solutions, we overlay our $\eta_{0}$

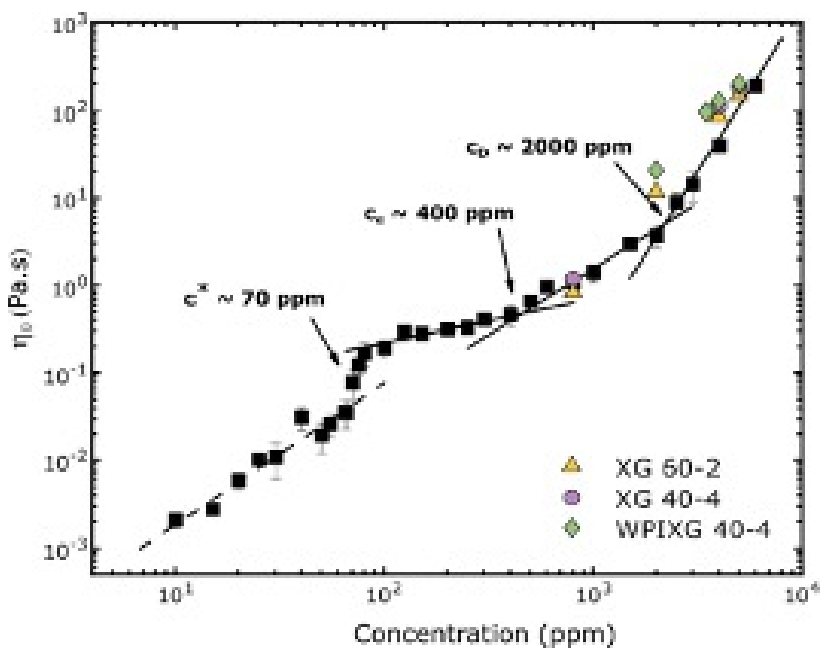

Figure 6: Superposition of zero-shear viscosity values over the $\eta_{0}$ vs concentration graph developed by Wyatt \& Liberatore [14] for several concentrations of salt-free xanthan gum solutions in water values over the data proposed by Wyatt \& Liberatore [14], where the $\eta_{0}$ is plotted against a wide range of concentrations for salt-free xanthan gum solutions (Figure 6). For concentrations (c) $\leq \mathrm{c}^{\star}$ the system is in the dilute regime. The region between $\mathrm{c}^{\star}$ and $\mathrm{c}_{e}$ corresponds to the semi dilute unentangled regime and concentrations between $\mathrm{c}_{e}$ and $\mathrm{c}_{d}$ belong to the semi dilute entangled regime. Finally, when concentrations are higher than $\mathrm{c}_{d}$, the system attains the concentrated regime. The superposition of our data over this graph clearly suggests that firstly, our data are very close in value and trend with respect to the curve of Wyatt \& Liberatore [14], and secondly that most of our solutions are in the concentrated regime.

\subsubsection{The second Newtonian plateau viscosity}

As stated before, the second Newtonian plateau has not received too much attention despite its interest in numerous industrial processes. In this study we proposed the use of the Huggins model [19], that is, when the system is highly diluted (equation 16). This could be the case in the high shear rate equilibrium since the molecules have completely different conformations and interactions, probably due to a much less hydrodynamic volume compared to the molecules at rest. To our knowledge, no research study has really addressed the study of the hydrodynamic volume of xanthan gum molecules in the second Newtonian plateau. The parameter $[\eta]_{\infty}$ is fitted to the data that follow a unique linear curve for XG solutions independently of the geometry used (Figure $5 \mathrm{~b}$ ), and the resulting value of $[\eta]_{\infty}=0.26$ confirms the former assumption. The global deviation is under 10\% (MAPD $\leq 10 \%)$.

In Figure 5b, it is observed that the presence of proteins is almost negligible at the second Newtonian plateau and a single model could be proposed for the three sets of data. This would indicate that the intrinsic viscosity of the xanthan gum molecules determines the viscosity at high shear rates to a greater extent than in the case of the viscosity at rest $\left(\eta_{0}\right)$. An additional study involving more concentrations would be necessary to support this view.

\subsubsection{The relaxation time and flow index}

The rheological parameters $m$ and $\lambda$ (Figure $5 c$ and $5 d$ ) are fitted empirically using adapted models. A power law model provides a good adjustment to $m$ values (MAPD $\leq$ $5 \%$ ) whereas an affine model gives a better fit for $\lambda$ values $(\mathrm{MAPD} \leq 5 \%)$. The model fitting parameters for these cases can be found in Table 3. For the variable $m$, the two models 
for XG solutions (60-2 and 40-4) are almost the same as expected. The model for the $m$ values for the WPIXG solutions presented the lowest error (MAPD $=1.02 \%$ ). This was probably due to the fact that these solutions were only characterized with the microdevice having a channel height of $50 \mu \mathrm{m}$ and not with the one with channel height of $150 \mu \mathrm{m}$. The latter is precisely the device providing data in the region where the two flow curves built in section 3.1 diverge $\left(\sim 10^{1}\right.$ to $\left.10^{3} \mathrm{~s}^{-1}\right)$. Therefore, not having data points in this diverging region is what might be accounting for lower error in the case of WPIXG solutions for the parameter $m$.

Concerning the higher errors for $\lambda$ for XG solutions when compared to WPIXG solutions, low-torque limit effects might be at the origin of this. According to the manufacturer the rotational rheometer used in this study has a minimum torque of $0.1 \mu \mathrm{N} \cdot \mathrm{m}$. As a result, for the less viscous solutions (XG008), data under a shear rate of $0.1 \mathrm{~s}^{1}$ might be less precise. Since $\lambda$ is determined by the transition between the $1^{\text {st }}$ Newtonian plateau and the shearthinning region (occurring precisely at around $0.1 \mathrm{~s}^{1}$ ), less precise $\lambda$ values for XG008 solutions might be contributing to the higher error identified for $\mathrm{XG}$ solutions.

\subsubsection{Synthesis of the rheological parameters}

In this section, the elements involved in the correlations of the four rheological parameters are recapitulated. In general, the models proposed described quite accurately the experimental data (MAPD $<10 \%)$. The most delicate case was the infinite-shear viscosity presenting the higher errors. However, acceptable values were obtained (MAPD in

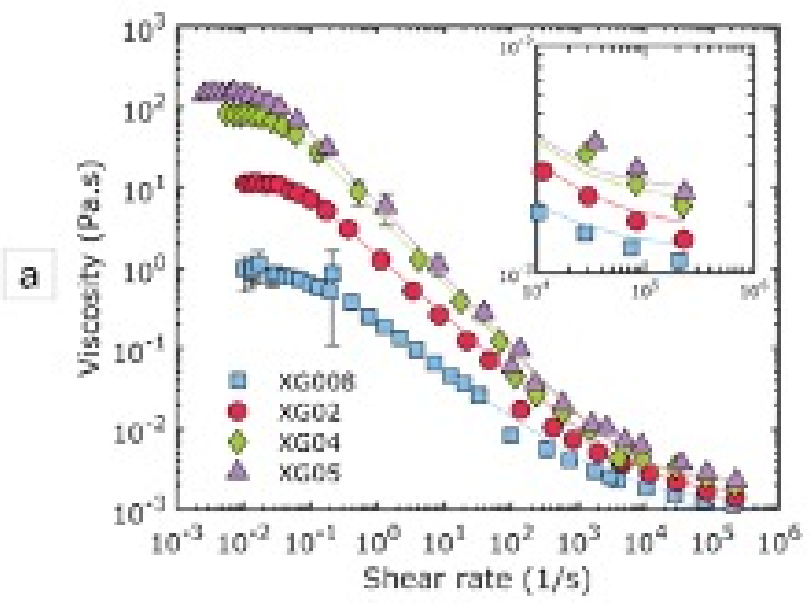

the range of 5 to 9\%). Renewed scientific interest and new technological advancements (such as microfluidic rheometry) will certainly help improve precision in measurements and fundamental understanding of molecular behavior at high shear rates.

\subsection{Evaluation of the quality of the correlations}

The correlations proposed in section 3.3 are now evaluated by using them to find the values of the different rheological parameters $\left(\eta_{0}, \eta_{\infty}, m\right.$ and $\left.\lambda\right)$ and then building a flow curve from these parameters (model fitting). Figure 7 presents the adjustment of these model fits (solid lines) to the experimental data (markers) for the XG solutions with the geometry 60-2 and for the WPIXG solutions with the geometry 40-4. The model fits adjust well the experimental data. However, some data around a shear rate range between 100 and $1000 \mathrm{~s}^{1}$ slightly deviate from the model fits. These points correspond to measurements performed with the microfluidic device having a greater depth of 150 $\mu \mathrm{m}$. The slight deviations are probably due to the minimal flow rate generated by the syringe pumps used with the microfluidic rheometer. To insure a very stable and a constant laminar flow of the fluids in the channel, with no mixing, no diffusion or turbulences during the co-flow even with miscible products, a minimum flow rate is set and by that the minimum shear rate provided is defined at $100 \mathrm{~s}^{-1}$. This technical limitation might lead to less precise determinations at low shear rates when using a microfluidic rheometer, thereby resulting in higher error in the model fits. A similar situation has been discussed Pipe

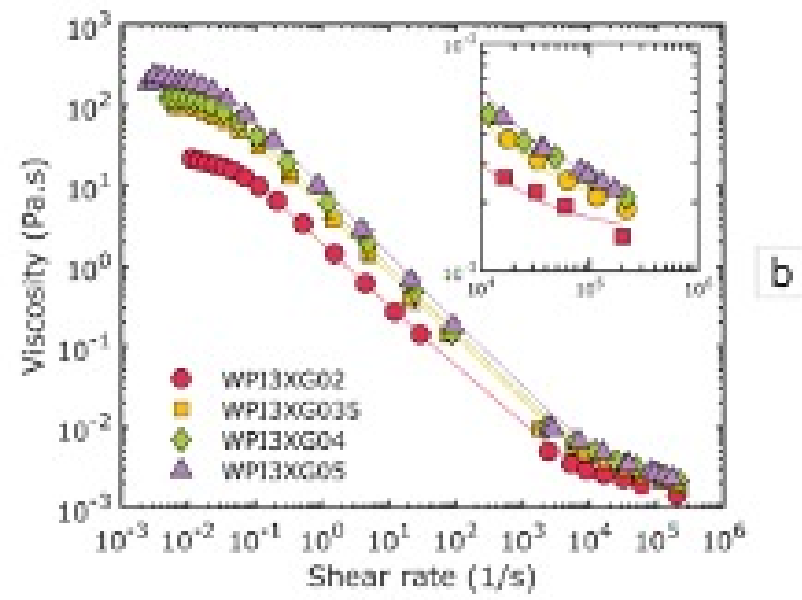

Figure 7: Experimental data and model fits (solid lines) built from the correlations as a function of concentration. a) XG solutions. b) WPIXG solutions 
Table 4: rheological variables of the model fits used in Figure 6 and mean absolute percent deviation between experimental data and model fit (MAPD)

\begin{tabular}{ccccccc}
\hline \multirow{2}{*}{ Solution } & Geometry & $\begin{array}{c}\eta_{0} \\
\mathrm{~Pa} \cdot \mathrm{s}\end{array}$ & $\begin{array}{c}\eta_{\infty} \\
\times 10^{-3} \mathrm{~Pa} \cdot \mathrm{s}\end{array}$ & $\begin{array}{c}\lambda \\
\mathrm{s}\end{array}$ & $\begin{array}{c}m \\
-\end{array}$ & $\begin{array}{c}\text { MAPD } \\
\%\end{array}$ \\
\hline XG008 & $60-2$ & 0.8 & 1.2 & 8.92 & 0.61 & 11.3 \\
XG02 & $60-2$ & 11.3 & 1.5 & 16.4 & 0.74 & 17.1 \\
XG04 & $60-2$ & 81.9 & 2.0 & 28.7 & 0.86 & 14.1 \\
XG05 & $60-2$ & 155 & 2.3 & 35 & 0.90 & 14.5 \\
WPI3XG02 & $40-4$ & 20.5 & 1.4 & 21.2 & 0.78 & 4.9 \\
WPI3XG035 & $40-4$ & 88.9 & 1.8 & 31.7 & 0.82 & 6.9 \\
WPI3XG04 & $40-4$ & 126 & 1.9 & 35.2 & 0.83 & 6.5 \\
WPI3XG05 & $40-4$ & 226 & 2.1 & 42.2 & 0.84 & 13 \\
\hline
\end{tabular}

et al. [36] for a xanthan gum solution with concentration $0.3 \%$ wt. Table 4 presents the rheological parameters calculated from the correlations proposed in section 3.3 that were used to plot the model fits presented in Figure 7 . The adjustment of the models to the experimental data is also presented in this table in terms of MAPD. The higher MAPD for the XG solutions is due precisely to the less precise fit at shear rates around 100-1000 $\mathrm{s}^{1}$ discussed previously. For the case of WPIXG solutions, since they were only characterized with the microdevice having a minor depth of 50 $\mu \mathrm{m}$, the values of MAPD tended to be lower.

\section{Conclusion}

The purpose of this study is to investigate a methodology to model the viscosity of xanthan gum-based solutions as a function of their concentration, over a large range of shear rates $\left(\sim 10^{-3}-10^{6} \mathrm{~s}^{-1}\right)$. More specifically, xanthan gum is a biopolymer widely encountered in industrial applications, so that many works have dealt with its rheology but few information concerning high shear rates is presented in the literature. This is why this work describes a complete methodology which is based on the analysis of experimental data obtained by combining two rheological techniques, rotational rheometry and microfluidic rheometry. In a first step, raw data obtained from the two techniques were analyzed and compared. The microfluidic data extend the rheometer data and the establishment of flow instabilities is determined at the shear rate where the two curves diverge. This point leads to the determination of a critical Reynolds number. Its value is linked to the coneplate geometry and is found to be lower when using a larger cone angle which is consistent with conclusions of previous works in the literature. A first limit in this subject is that no generalized Reynolds or Taylor number defini- tion has been found to give a unique critical value for the different cone-plate geometries.

At the lowest shear rates attained by the microfluidic rheometer, a slight discrepancy with the rotational rheometer is observed which is due to the difficulty of getting an accurate and stable fluid flow. By merging the results from the two rheometers, a relevant flow curve can be established over the whole shear rate range by using a Williams-Carreau equation. The four parameters from the Williams-Carreau law are modeled as a function of polymer concentration. The first Newtonian plateau is in good agreement with literature data in the considered dilution regime. The second Newtonian plateau, which is the most sensitive parameter for our purpose of viscosity model implementation in high shear processes, is also the most difficult to determine accurately. Thanks to the Fluidicam ${ }^{\mathrm{TM}}$ device, it is now possible to have a realistic model for the concentration range of interest, with a sufficient accuracy for the needs of process engineering. The findings from this research might benefit applications in fields of food, cosmetics, coatings and other related sectors. Additionally, this study contributs to the understanding of rheology at high shear rates for a polymer mixture (xanthan gum with whey protein isolates) of industrial relevance for food applications.

Acknowledgement: This work was carried out with the financial support of the regional program "Food for Tomorrow / Cap Aliment; Research, Education and Innovation in Pays de la Loire", which is supported by the French Region Pays de la Loire and the European Regional Development Fund (FEDER).

Conflict of Interest: Authors state no conflict of interest. 


\section{References}

[1] Turian RM. Perturbation Solution of the Steady Newtonian Flow in the Cone and Plate and Parallel Plate Systems. Ind Eng Chem Fundam. 1972 Aug;11(3):361-8.

[2] Davies GA, Stokes JR. Thin film and high shear rheology of multiphase complex fluids. J Non-Newton Fluid Mech. 2008 Jan;148(13):73-87.

[3] Garcıá-Ochoa F, Santos VE, Casas JA, Gómez E. Xanthan gum: production, recovery, and properties. Biotechnol Adv. 2000 Nov;18(7):549-79.

[4] Sworn G. Xanthan gum. In: Phillips GO, Williams PA, editors. Handbook of hydrocolloids. 2. ed. Boca Raton, Fla.: CRC; 2009. p. 186-203. (Woodhead Publishing in food science, technology and nutrition).

[5] Giboreau A, Cuvelier G, Launay B. Rheological behaviour of three biopolymer/water systems, with emphasis on yield stress and viscoelastic properties. J Texture Stud. 1994 Jun;25(2):119-38.

[6] Tecante A. Viscoelasticity. In: Barbosa-Cánovas GV, editor. Food Engineering [Internet]. Paris; EOLSS: UNESCO; 2005 [cited 2019 Jun 28]. p. 285-303. (Encyclopedia of life support sys tems.). Available from: http://catalog.hathitrust.org/api/volu mes/oclc/64183236.html

[7] Van Aken G. Polysaccharides in Food Emulsions. In: Food polysaccharides and their applications. 2nd ed. Boca Raton, FL: CRC/Taylor \& Francis; 2009. p. 521-39. (Food science and technology).

[8] Rodd AB, Dunstan DE, Boger DV. Characterisation of xanthan gum solutions using dynamic light scattering and rheology. Carbohydr Polym. 2000 Jun;42(2):159-74.

[9] McClements DJ. Food emulsions: principles, practices, and techniques. 2. ed. Boca Raton, Fla.: CRC Press; 2005. 609 p. (CRC series in contemporary food science).

[10] Coviello T, Kajiwara K, Burchard W, Dentini M, Crescenzi V. Solution properties of xanthan. 1. Dynamic and static light scattering from native and modified xanthans in dilute solutions. Macromolecules. 1986 Nov;19(11):2826-31.

[11] Gu Z. Experimental and Theoretical Study of Droplet Formation at a T-junction with Xanthan Gum Solutions [Thesis]. University of New South Wales; 2013.

[12] Southwick JG, Jamieson AM, Blackwell J. Quasi-elastic light scattering studies of semidilute xanthan solutions. Macromolecules. 1981 Nov;14(6):1728-32.

[13] Milas M, Rinaudo M, Knipper M, Schuppiser JL. Flow and viscoelastic properties of xanthan gum solutions. Macromolecules. 1990 Apr;23(9):2506-11.

[14] Wyatt NB, Liberatore MW. Rheology and viscosity scaling of the polyelectrolyte xanthan gum. J Appl Polym Sci. 2009 Dec 15;114(6):4076-84

[15] Roy A. Synthèse et caractérisation de dérivés amphiphiles du xanthane [Thesis]. Université du Havre; 2015.

[16] Utracki L, Simha R. Corresponding state relations for the viscosity of moderately concentrated polymer solutions. J Polym Sci A. 1963 Apr;1(4):1089-98.

[17] Rao MA. Food rheology and texture. In: Barbosa-Cánovas GV, editor. Food Engineering. Paris, France: UNESCO; 2005. p. 253-

[18] Khouryieh HA, Herald TJ, Aramouni F, Alavi S. Intrinsic viscosity and viscoelastic properties of xanthan/guar mixtures in dilute so- lutions: Effect of salt concentration on the polymer interactions. Food Res Int. 2007 Aug;40(7):883-93.

[19] Huggins ML. The Viscosity of Dilute Solutions of Long-Chain Molecules. IV. Dependence on Concentration. J Am Chem Soc. 1942 Nov;64(11):2716-8.

[20] Kraemer EO. Molecular Weights of Celluloses and Cellulose Derivates. Ind Eng Chem. 1938 Oct;30(10):1200-3.

[21] Schulz GV, Blaschke F. Eine Gleichung zur Berechnung der Viscositätszahl für sehr kleine Konzentrationen, [Molekulargewichtsbestimmungen an makromolekularen Stoffen, IX]. J Für Prakt Chem. 1941 Mar 22;158(1-8):130-5.

[22] Tanglertpaibul T, Rao MA. Intrinsic Viscosity of Tomato Serum as Affected by Methods of Determination and Methods of Processing Concentrates. J Food Sci. 1987 Nov;52(6):1642-5.

[23] Chou TD, Kokini JL. Rheological Properties and Conformation of Tomato Paste Pectins, Citrus and Apple Pectins. J Food Sci. 1987 Nov;52(6):1658-64.

[24] Larson RG. Instabilities in viscoelastic flows. Rheol Acta. 1992;31(3):213-63.

[25] Gupta S, Wang WS, Vanapalli SA. Microfluidic viscometers for shear rheology of complex fluids and biofluids. Biomicrofluidics. 2016 Jul;10(4):043402.

[26] Ewoldt RH, Johnston MT, Caretta LM. Experimental Challenges of Shear Rheology: How to Avoid Bad Data. In: Spagnolie SE, editor. Complex Fluids in Biological Systems: Experiment, Theory, and Computation [Internet]. New York, NY: Springer New York; 2015 [cited 2019 Jul 25]. p. 207-41. (Biological and Medical Physics, Biomedical Engineering). Available from: https: //doi.org/10.1007/978-1-4939-2065-5_6

[27] Paul EL, Atiemo-Obeng VA, Kresta SM, editors. Handbook of industrial mixing: science and practice. Hoboken, N.J: WileyInterscience; 2004. 1377 p.

[28] Rao MA. Application of rheology to fluid food handling and processing. In: Rao MA, editor. Rheology of fluid and semisolid foods: principles and applications. 2nd ed. New York: Springer; 2007. p. 427-69. (Food engineering series).

[29] Kang K, Lee LJ, Koelling KW. High shear microfluidics and its application in rheological measurement. Exp Fluids. 2005 Feb;38(2):222-32.

[30] Pan L. Complex Fluids in Microchannel Flows at Low Reynolds Number: Elastic Instabilities and Rheology [Thesis]. [Pennsylvania, USA]: University of Pennsylvania; 2013.

[31] Sepulveda J, Montillet A, Della Valle D, Loisel C, Riaublanc A. Deformation of gas-liquid interfaces in a non-Newtonian fluid at high throughputs inside a microfluidic device and effect of an expansion on bubble breakup mechanisms. Chem Eng Sci. 2020 Feb;213:115377.

[32] Sharp KV, Adrian RJ, Santiago JG, Molho JI. Liquid Flows in Microchannels. In: Gad-el-Hak M, editor. MEMS: introduction and fundamentals. 2nd ed. Boca Raton, Florida, USA: CRC/Taylor \& Francis; 2006

[33] Nghe P, Terriac E, Schneider M, Li ZZ, Cloitre M, Abecassis B, et al. Microfluidics and complex fluids. Lab Chip. 2011;11(5):788.

[34] Guillot P, Panizza P, Salmon J-B, Joanicot M, Colin A, Bruneau C-H, et al. Viscosimeter on a Microfluidic Chip. Langmuir. 2006 Jul;22(14):6438-45.

[35] Pan L, Arratia PE. A high-shear, low Reynolds number microfluidic rheometer. Microfluid Nanofluidics. 2013 May;14(5):885-94.

[36] Pipe CJ, Majmudar TS, McKinley GH. High shear rate viscometry. Rheol Acta. 2008 Jul;47(5-6):621-42. 
[37] Comba S, Dalmazzo D, Santagata E, Sethi R. Rheological characterization of xanthan suspensions of nanoscale iron for injection in porous media. J Hazard Mater. 2011 Jan;185(2-3):598-605.

[38] Xu F, Midoux N, Li H-Z, Hébrard G, Dietrich N. Characterization of Bubble Shapes in Non-Newtonian Fluids by Parametric Equations. Chem Eng Technol. 2019 Nov;42(11):2321-30.

[39] Mrokowska MM, Krztoń-Maziopa A. Viscoelastic and shearthinning effects of aqueous exopolymer solution on disk and sphere settling. Sci Rep. 2019 Dec;9(1):7897.

[40] Dakhil H, Wierscheim A. Measuring low viscosities and high shear rates with a rotational rheometer in a thingap parallel-disk configuration. Appl Rheol [Internet]. 2014 [cited 2021 Feb 18]; Available from: http://www.ar.ethz.ch/cgibin/AR/view?DOI=10.3933/ApplRheol-24-63795

[41] Connelly RW, Greener J. High-Shear Viscometry with a Rotational Parallel-Disk Device. J Rheol. 1985 Apr;29(2):209-26.

[42] Dontula P, Macosko CW, Scriven LE. Does the Viscosity of Glycerin Fall at High Shear Rates? Ind Eng Chem Res. 1999 Apr;38(4):172935.

[43] Miller C. Predicting Non-Newtonian Flow Behavior in Ducts of Unusual Cross Section. Ind Eng Chem Fundam. 1972 Nov;11(4):5248.

[44] Macosko CW. Rheology: principles, measurements, and applications. New York: VCH; 1994. 550 p. (Advances in interfacial engineering series).

[45] Fewell ME, Hellums JD. The Secondary Flow of Newtonian Fluids in Cone-and-Plate Viscometers. Trans Soc Rheol. 1977 Dec;21(4):535-65.

[46] Cheng DC-H. The effect of secondary flow on the viscosity measurement using the cone-and-plate viscometer. Chem Eng Sci. 1968 Aug;23(8):895-9.

[47] Chaturani P, Narasimman S. Flow of power-law fluids in coneplate viscometer. Acta Mech. 1990 Sep;82(3-4):197-211.

[48] Delaplace G, Guérin R. Mélange des produits pâteux - Caractéristiques d'un système agité. Tech Ing Opérations Unit Génie Ind Aliment [Internet]. 2006; base documentaire: TIB430DUO.(ref. article: f3350). Available from: https://www.techniques-ingenieur. $\mathrm{fr} /$ base-documentaire/procedes-chimie-bio-agro-th2/operatio ns-unitaires-du-genie-industriel-alimentaire-42430210/melan ge-des-produits-pateux-f3350/
[49] Heuser G, Krause E. The flow field of Newtonian fluids in cone and plate viscometers with small gap angles. Rheol Acta. 1979 Sep;18(5):553-64.

[50] Sdougos HP, Bussolari SR, Dewey CF. Secondary flow and turbulence in a cone-and-plate device. J Fluid Mech. 1984 Jan;138:379404.

[51] Kulicke WM, Porter RS. Irregularities in steady flow for nonnewtonian fluids between cone and plate. J Appl Polym Sci. 1979 Feb 15;23(4):953-65.

[52] Amundarain JL, Castro LJ, Rojas MR, Siquier S, Ramírez N, Müller AJ, et al. Solutions of xanthan gum/guar gum mixtures: shear rheology, porous media flow, and solids transport in annular flow. Rheol Acta. 2009 Jun;48(5):491-8.

[53] Narchi I, Vial Ch, Djelveh G. Effect of protein-polysaccharide mixtures on the continuous manufacturing of foamed food products. Food Hydrocoll. 2009 Jan;23(1):188-201.

[54] Pelletier E, Viebke C, Meadows J, Williams PA. A rheological study of the order-disorder conformational transition of xanthan gum. Biopolymers. 2001;59(5):339-46.

[55] Whitcomb PJ, Macosko CW. Rheology of Xanthan Gum. J Rheol. 1978 Oct;22(5):493-505.

[56] Benichou A, Aserin A, Lutz R, Garti N. Formation and characterization of amphiphilic conjugates of whey protein isolate (WPI)/xanthan to improve surface activity. Food Hydrocoll. 2007 May;21(3):379-91.

[57] Cheftel JC, Lorient $D$. Les propriétés fonctionnelles des protéines laitières et leur amélioration. Le Lait. 1982;62(617-620):435-83.

[58] Wijayanti HB, Bansal N, Deeth HC. Stability of Whey Proteins during Thermal Processing: A Review: Thermal stability of whey proteins... Compr Rev Food Sci Food Saf. 2014 Nov;13(6):123551.

[59] Kamykowski GW. Materials Characterization by Rheological Methods [Internet]. 2019 [cited 2020 Nov 6]. Available from: https://www.tainstruments.com/wp-content/uploads/201904-AM-Rheology.pdf 